\title{
Société Suisse de Cardiologie
}

\section{Seance du 8 novembre 1959 à Bale}

Verzeichnis der Vortrage - Lisle des communications

Widmer, L. K.; Capeller, D. von und Staub, H. (Basel): Mus-

keldurchblutung und inter mi ttierende venose Stauung . 194

Schelling, J.-L.; Reymond, Cl. et Rívíer, J.-L. (Lausanne):

Intérêt de Tenregistrement simultané du phonocardiogramme et du cardiogramme apexien 199

Lüthy, E.; Rösli, R. und Bíschof, B. (Zurich): Das Verhalten des Herzglykogens bei normalen und hypertrophierenden Rattenherzen nach ACTH und STH . . . . . .209

Reymond, CL; Rossel, P.-A. et Rívíer, J.-L. (Lausanne): Traitement à Tiproniazide et métabolisme intermédiaire des hydrates de carbone $\quad 21<5$

Holzinann, M. (Zurich): Beitrag zur Kenntnis der VorhofParasystolie 223

Gillmann, H. und Löhr, B. (Düsseldorf): Vergleichsuntersuchungen zwischen Elektrokardiogramm und Operationsbefund bei Kammerseptumdefekten 232 Rutíshauser, W.; Lüthy, E. und Hegglin, R. (Zurich): Zur oxymetrischen Differenzierung zwischen links-rechts-Shunt und Klappeninsuffizienz des linken Herzens . . 242

Schmid, A. (Bern): Folgerungen aus einer Beziehung zwi schen Periodik und Höhe des diaslolischen Blutdruckes hinsichtlich langfristigen Druckverhaltens bei NephrilisPatienten 249

Cardiologia, Vol. 36, No. 4 (I960) 